Original Research Paper

\title{
Survey on Early Detection of Alzhiemer's Disease Using Capsule Neural Network
}

\author{
Sharunya S. $\mathbf{R}^{1}$, Vijayalakshmi Desai ${ }^{1}$, Meenakshi Singh ${ }^{1}$, Kusuma Mohanchandra ${ }^{1 *}$ \\ ${ }^{1}$ Department of Information Science and Engineering. Dayananda Sagar Academy of \\ Technology and Management, Bangalore, Karnataka, India.
}

Article History
Received:
28.02 .2020
Revised:
25.03 .2020
Accepted:
24.04 .2020
*Corresponding Author:
Kusuma Mohanchandra
Email:
kusumalak@gmail.com

This is an open access article, licensed under: $\mathrm{CC}-\mathrm{BY}-\mathrm{SA}$

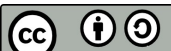

Abstract: Alzheimer's disease (AD) is a disorder which is irreversible of the brain related to memory loss, mostly found in the old and aged population. Alzheimer's dementia results from the degeneration or loss of brain cells. The brain-imaging technologies most often used to diagnose AD is Magnetic resonance imaging (MRI). MRI or structural magnetic resonance is a very popular and actual technique used to diagnose AD. An MRI uses magnets and powerful radio waves to create a complete view of your brain. To actually detect the presence of Alzheimer's, the MRI should me studied carefullyImplementation of CBIR Content Based Image Retrival which is a revolutionary computer aided diagnosis technique will create new abilities in MRI Magnetic resonance imaging in related image retrieval and training for recognition of development of $\mathrm{AD}$ in early stages.

Keywords: Alzheimer's Disease, Content Based Image Retrieval, Magnetic Resonance Imaging. 


\section{Introduction}

Alzheimer's disease is a common cause of a degenerative brain disease, dementia which is characterized by a decline in memory, language, problem solving and other cognitive skills. Alzheimer's disease affects a person's ability to perform everyday activities [1] [2] [3] [4]. It is a neurodegenerative disease, characterized by progressive impairment of neurons and synaptic functioning affecting more than 47 million of people worldwide [5] [6] [7].

Alzheimer's disease was first identified more than 100 years ago, but after almost 70 years, it was recognized as "major killer", the most common cause of dementia. In the year 2006, 26600 thousand people suffered from this disease worldwide. Alzheimer's disease is predicted to globally affect 1 in 85 people [8] that is an estimate of 115400 thousand people will suffer from AD in 2050, and at least $43 \%$ of prevalent cases need high level of care which can result in overwhelming consequences in terms of healthcare expenses and quality of life of patients and caregivers. Increasing life expectancy in devel oped countries has led to growth in number of cases of people affected by age-related neurodegenerative diseases, such as Alzheimer's disease (AD) [9].

Researchers have revealed ample information about Alzheimer's, yet exact biologic changes that cause Alzheimer's, reason for quick progress in a few compared to others and how the disease can be prevented, decelerated or stopped, is about to be discovered. AD affects people over 65 years of age, while early-onset Alzheimer's disease is diagnosed before 65 years [10]. Even though some medical treatment may temporarily show encouraging effects, nothing has demonstrated the capability of preventing deterioration. People with mild cognitive impairment (MCI) are at the transitional stage from normal control $(\mathrm{NC})$ to $\mathrm{AD}$, and they might have a very high risk of deteriorating to dementia especially the AD type [11].

The current stage in the development of a product of clinical diagnosis of $\mathrm{AD}$ requires a specialty clinic and includes a medical examination, neuropsychological testing, neuroimaging, cerebrospinal fluid (CSF) analysis and blood examination. The above process is neither cost nor time-effective. Furthermore, given the rapidly aging worldwide population with a predictable dramatic increase of $\mathrm{AD}$ cases, there are inadequate numbers of specialty clinics and hospitals to meet the budding needs [12]. Plentiful effort has been expended in this direction, resulting in a variety of methods or approaches for early Alzheimer's disease detection with MRI data. Those approaches address the extraction of discriminative features, the selection of the most apt features from a large pool of features, and the selection of efficient classification models from machine learning and computational intelligenc [13].

\section{Survey Study}

Yao, et al [2] proposes a process by converting the 4-way classification into five binary classification problems which is a hierarchical process. A feature selection technology which is new was also proposed which identifies more informative and brief subset from 3 demographic features and $426 \mathrm{~s}$ MRI morphometric, to ensure that highest accuracy is achieved by each binary classifier. The method gives a new framework using hierarchical grouping for multi way classification and accurate feature selection.

While Liu, et al [3] uses MRI images to detect the Alzheimer's disease and also developing stage where MCI (Mild Cognitive Impairment) gets converted to Alzheimer's disease. The whole brain is of four sections named as 90, 54, 14 and 1 regions divided based on AAL (Automated Anatomical Labelling). For data pre-processing Pearson correlation is taken and for the feature selection F-scores was used to lessen the dimensionality of features. In final stage, MKBoost (Multiple Kernel Boosting) algorithm for classification of the disease from the healthy control (HCs).

Amoroso, et al [4] used Random Forest feature selection and Deep Neural Network classification and presented a classification strategy using a mixed unit including the four classes of classification problem, I,e HC, AD, MCI, cMCI, for training the model. Also, they compared this method with an original classification strategy based on fuzzy logic learned on a mixed unit including only Healthy Control and Alzheimer's disease. Actually, they intend to examine if DNN accuracy can be better after 344 combining different imaging modalities or by adding non-MRI images.

Lie, et al [5] was the first on the basis of the availability of different modalities divide the actual data into multiple views and then they build a hypergraph in each view space on the basis of sparse representation. VAHC (View Aligned Hypergraph Classification) model is then given, based on viewaligned regularize to capture consistency among all the views. They then collect the class probability 
scores produced from VAHC, by using a multi-view label fusion method used for making a final classification decision. They assessed their method on the baseline ADNI-1 database with 807 themes and three modalities which are MRI, PET, and CSF. The results prove that their method beats stateof-the-art approach that use unfinished multi-modality data for AD/MCI diagnosis.

Jun Zhang, et al 6] uses Magnetic Resonance Images (MRI) images for the early detection of AD using $\mathrm{AD}$ landmarks for distinguishing $\mathrm{AD}$ subjects from Healthy control in the first phase nonlinear registration subjects and the second step with the brain tissue segmentation. For pre-processing the data voxel wise comparisons is done on the datasets. For feature selection they have used random forest. The morphological features were extracted from the AD landmarks which was identified for training the SVM (Support Vector Machine) classifier using feature selection.

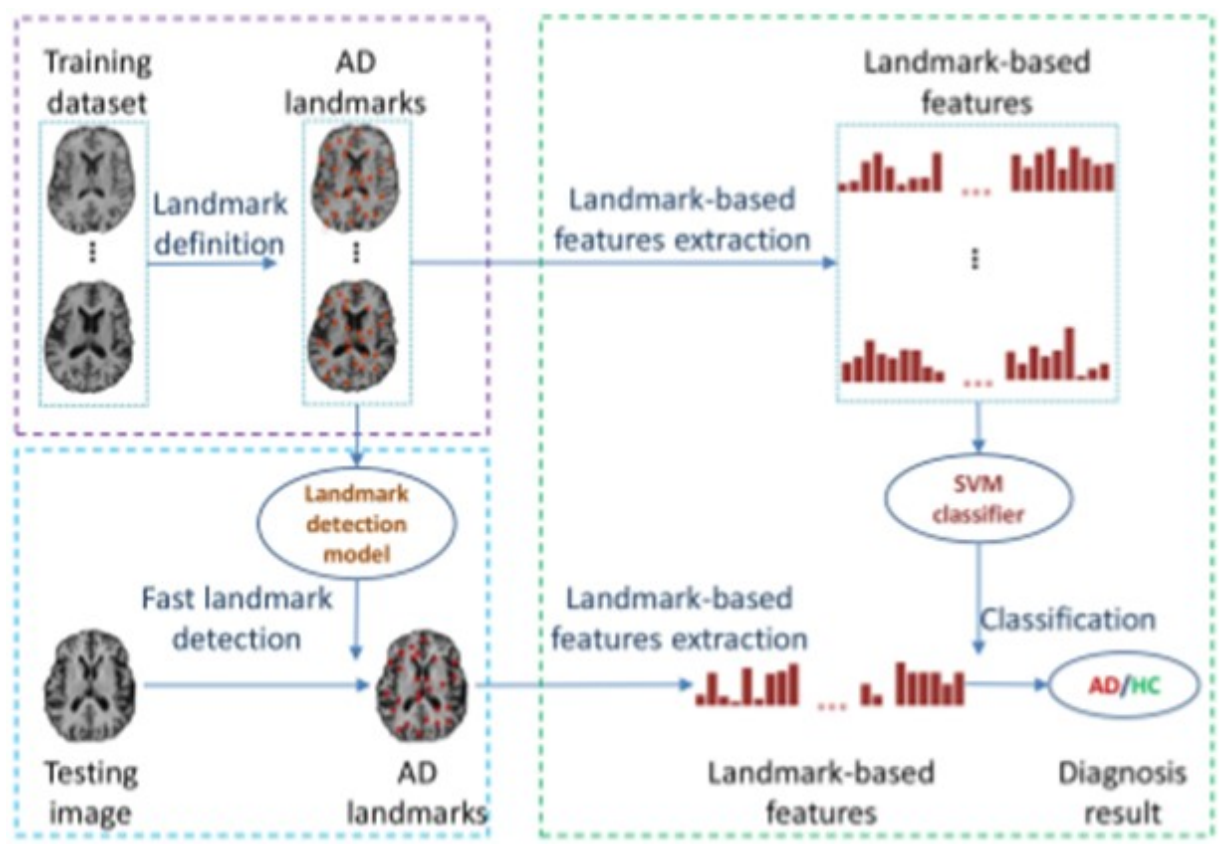

Figure 1. Diagram Illustrating the Steps in the Proposed Landmark Detection and AD Classification Framework

In general, the proposed framework defines three sequential steps:

1. Landmark definition

2. Landmark Detection

3. $\mathrm{AD} / \mathrm{HC}$ Classification

Nanni, et al [7] gave a technique to perform early diagnosis of AD, on both MRI images and blood plasma proteins expression values, combining different feature reduction approaches. SVM (Support vector machine) is trained by Every selected set of features, then the weighted sum rule is used to combined set of SVMs. SVM is basically trained with the feature vector of proposed images where the images and used to train an SVM are quarried from the image.

Kruthikaa, et al [14] uses all the MRI images to detect the Alzheimer's disease using the Content based image retrieval (CBIR) techniques. The data-pre-processing of data is done using the software known as statistical parametric (SPM) version 1.2 and for learning and training process sparse autoencoder. The final stage for classification capsule network is used for differentiating Alzheimer's from healthy control. 
$\mathrm{Ju}$, et al [15] used deep learning with brain network and few other related information to make early analysis of $\mathrm{AD}$. The other related information include gender, age and $A p o E$ gene of the subject. Computing the functional connectivity of brain regions using R-fMRI (Resting - State Functional Magnetic Resonance Imaging) data, facilitates construction of the brain network. Aging and MCI, an early stage of $\mathrm{AD}$, is distinguished by building a targeted autoencoder network chwhi provides a dependable classifier for Alzheimer's detection.

Yue, et. al. [16] employed DCNN depp convolutional neural network to obtain the most suitable features from the MRI images of patients. The MRI images are pre-processed in a stern pipeline manner at first. Then, each volume is re-sliced, and put the same into a DCNN directly. At last 4 different stages of $\mathrm{AD}$ are known.

\section{Methodology}

Yao et. al. [2] used a 4 different classification for predicting alzheimer's disease and Mild Cognitive Impairment (MCI) happens in different ages of people. The 4-way classification is classified into $\mathrm{AD}, \mathrm{MCI}, \mathrm{cMCI}$ and healthy control. The machine learning approaches are imposed for preprocessing the data which was taken from Kaggle hosted by a competition, on the T1-weighted MRI images data and information from the International Alzheimer's Disease Neuroimaging (ADNI) database. There are some calculated scores for virtual data like MMSE which specifies that it may loose some useful information like p-Values on virtual data which do not exist momentous difference. For the feature selection, different traditional methods are compared with the algorithms like Forward selection and SVM-FRE which was gave number of features, accuracy, sensitivity and specificity. Forward selection is a type of regression which begins with an empty model and adds all variables one by one. In each forwarded step, it add one variable that gives the single best improvement to the model. Then for the binary classification SVM defined as supervise machine learning algorithm which can be used for classification or regression problems. It uses different technique called the KT (Kernel Trick) to transform data and then based on these transformations it finds an optimum boundary between the possible outputs.54.38\% accuracy which was achieved with the 4-way classification on the testing data.

Nanni, et. al. [7] uses brain MRI studies for the early recognition of the Alzheimer's disease. For the feature selection approaches like Fisher Scores (based on different methods), Gini Index (an arithmetical measure of dispersion), T-test (based on different student's distribution), Sparse multinomial logical regression via Bayesian L1 regularization and so many. For the classification purpose convolutional neural network is used for reshaping the vector into a matrix methods which are used like Triplet, Continuous Wavelet, Random reshaping and Gabor filter feature extraction. Triplet is defined by highlighted vector that describes a pattern which is then regularized to a vector. Random reshaping is randomly sorting the original vector which can be fed to the SVM which is next used for the classification between different classification OD detection between AD vs MCI and others. The accuracy of $92.4 \%$ for $\mathrm{AD}$ and $\mathrm{CN}, 85.5 \%$ for MCIc and $\mathrm{CN}, 67.5 \%$ for MCIc and MCInc and $96.8 \%$ for MRI after using Fisher method.

Yue, et. al. [16] uses to analyse the Alzheimer's with many symptoms such as weakening of memory and language on ages. Traditional methods of diagnosing $\mathrm{AD}$ and mild cognitive impairment non-parametric non uniform bias correction (N3 algorithm), both are done with grinder pipeline software. A machine learning pipeline are used to help automate machine learning workflows and algorithms. They operate by empowering a sequence of data to be transformed and associated together in a model that can be tested and assessed to achieve an outcome, whether it is positive or negative. Then brain extraction is used with FSL-BET toolbox, FSL is an inclusive library of analysis tools for FMRI, MRI and DTI brain imaging data. Most of the tools can be track both from the command line and as GUIs (Graphical User Interfaces). The features extracted with the grey matter (gm), White Matter (wm).The method for the classification such as SVM which gave accuracy of $81.04 \%$ for NC vs AD, $76.98 \%$ for NC vs MCI and $70.15 \%$ for AD vs MCI. Convolutional Neural Network (CNN) results with the accuracy of $95.441 \%$ for $\mathrm{NC}$ and $\mathrm{AD}, 95.38 \%$ for $\mathrm{NC}$ and $\mathrm{MCI}$ and $93.88 \% \mathrm{AD}$ and MCI.

Jin Liu, et. al. [3] uses different region of interest for analysis of brain magnetic resonance imaging to predict the disease and also the initial developing stage where mild congnitive impairment (MCI) transformed into AD. The whole brain parts are divided into 4 sections names as 90, 54, 14 and 1 zones differentiated based on the automated anatomical labelling. For data pre- 
processing Pearson correlation is taken after which for the feature selection F-scores was used to reduce the dimensions of features and for the feature ranking. So, the greater the F-scores, area more likely that the feature will be discriminative. The final stage is multiple kernel boosting algorithm for the classification. Multiple Kernel Boosting (MKboost) algorithm is a machine learning algorithm which has attracted many attentions in recent years and for the SVM toolbox, LBSVM are used. It is an open source machine learning library written in c++ and used c API. The classification results with accuracy of $94.65 \%$.

Kruthikaa \& Maheshappa [14] uses all the MRI images to predict the Alzheimer's disease using the Content Based Image Retrieval (CBIR) techniques. The data pre-processing are done with the software known as statistical parametric (SPM) version 1.2. For learning and training purpose sparse autoencoder had been used. The final stage of classification, capsule networks are used for differentiating alzheimer's from healthy control. Skull scraping and normalizing had ensure the compatibility between images after transforming original brain images into standard image space. Using autoencoder which is a 3-layered artificial neural network, basically used for the unsupervised data to differentiate between different kinds of data.

\section{Result Comparison}

Results of all the papers [7] [4] [16] are tabulated in Table 1, for comparing the results.

Tabel 1. Comparison the Result of the Papers [7] [4] [16]

\begin{tabular}{|c|c|c|c|c|c|}
\hline PAPER & & МЕТНОI & AND ACCU & $\mathrm{CY}$ & \\
\hline \multirow{5}{*}{ [7] } & METHOD & ADvs CN & $\begin{array}{c}\text { MCIc vs } \\
\text { CN }\end{array}$ & $\begin{array}{c}\text { MCIc } \\
\text { vsMCInc }\end{array}$ & MRI \\
\hline & Gini & 85.6 & 80.5 & 63.2 & 82.4 \\
\hline & $\mathrm{Fi}$ & 92.4 & 85.5 & 67.5 & 96.8 \\
\hline & SBMLR & 91.5 & 83.3 & 68 & 94.7 \\
\hline & Ttest & 91 & 82.2 & 64.2 & 95.2 \\
\hline \multirow[b]{2}{*}{ [14] } & METHOD & $\mathrm{MCI}$ vs $\mathrm{AD}$ & $\mathrm{NC}$ vs $\mathrm{AD}$ & $\mathrm{NC}$ vs MCI & \\
\hline & $\begin{array}{c}\text { CAPSULE } \\
\text { NETWORK }\end{array}$ & 94.6 & 92.8 & 94.04 & \\
\hline \multirow{4}{*}{ [16] } & METHODS & NCvs AD & $\mathrm{NC}$ vs MCI & $\mathrm{AD}$ vs $\mathrm{MCI}$ & \\
\hline & $\mathrm{CNN}$ & 95.441 & 95.388 & 93.888 & \\
\hline & SVM & 81.04 & 76.98 & 70.15 & \\
\hline & SAE & 91.65 & 87.59 & 77.27 & \\
\hline
\end{tabular}

\section{Conclusion}

From all the above papers we have discussed in the above sections we have come to the conclusion that only 4 papers are more relevant to our project. We are going to use the MRI(magnetic Resonance Images) as a data set, to detect the Alzheimer's disease and also developing stage where Mild Cognitive Impairment (MCI) gets converted to AD from the Classification of Alzheimer's disease using whole brain hierarchical network paper, MKBoost (Multiple Kernel Boost) algorithm for classification of the disease from the Healthy Control (HCs) and from the paper 3 they use all the MRI images to detect the AD using the Content Based Image Retrieval (CBIR) techniques. We have come to the conclusion that from paper1 CNN method can be used as it has the highest accuracy than the other algorithms used in the paper. Paper has the most accuracy for SBMLR method therefore it can be used in the paper. 


\section{References}

[1] Anonymous, "Alzheimer's disease facts and figures," Alzheimer's \& dementia: the journal of the Alzheimer's Association, vol. 11, no. 3, pp. 332, 2015.

[2] D. Yao, V. D. Calhoun, Z. Fu, Y. Du, and J. Sui, "An ensemble learning system for a 4-way classification of Alzheimer's disease and mild cognitive impairment," Journal of neuroscience methods, vol. 302, pp. 75-81, 2018.

[3] J. Liu, M. Li, W. Lan, F.X. Wu, Y. Pan, and J. Wang, "Classification of Alzheimer's disease using whole brain hierarchical network," IEEE/ACM transactions on computational biology and bioinformatics, vol. 15, no. 2, pp. 624 - 632, 2016.

[4] N. Amoroso, D. Diacono, A. Fanizzi, M. La Rocca, A. Monaco, A. Lombardi, C. Guaragnella, R. Bellotti, S. Tangaro, and Alzheimer's "Disease Neuroimaging Initiative, 2018 Deep learning reveals Alzheimer's disease onset in MCI subjects: results from an international challenge," Journal of neuroscience methods, vol. 302, pp. 3-9, 2018.

[5] M., Liu, J. Zhang, P. T. Yap, and D., Shen, "View-aligned hypergraph learning for Alzheimer's disease diagnosis with incomplete multi-modality data," Medical image analysis, 36, pp.123 134, 2017.

[6] J. Zhang, Y. Gao, Y. Gao, B. C. Munsell, and D. Shen, "Detecting anatomical landmarks for fast Alzheimer's disease diagnosis," IEEE transactions on medical imaging, vol. 35, no. 12, pp. 2524-2533, 2016.

[7] L. Nanni, C. Salvatore, A. Cerasa, and I. Castiglioni, "Combining multiple approaches for the early diagnosis of Alzheimer's Disease," Pattern Recognition Letters, vol. 84, pp. 259 - 266, 2016.

[8] Y. Zhang, S. Wang, P. Phillips, Z. Dong, G. Ji, and J. Yang, "Detection of Alzheimer's disease and mild cognitive impairment based on structural volumetric MR images using 3D-DWT and WTA-KSVM trained by PSOTVAC," Biomedical Signal Processing and Control, vol. 21, pp. 58-73, 2015.

[9] A. Aramendi, A. Weakley, M. Schmitter-Edgecombe, D. J. Cook, A. Aztiria Goenaga, A. Basarab, and M. Barrenechea Carrasco, "Smart home-based prediction of multi-domain symptoms related to Alzheimer's disease," IEEE Journal of Biomedical and Health Informatics, 2018.

[10] R. K. Lama, J. Gwak, J. S. Park, and S.W. Lee, "Diagnosis of Alzheimer's disease based on structural MRI images using a regularized extreme learning machine and PCA features," Journal of healthcare engineering, 2017.

[11] N. Zeng, H. Qiu, Z. Wang, W. Liu, H. Zhang, and Y. Li, "A new switching-delayed-PSO-based optimized SVM algorithm for diagnosis of Alzheimer's disease," Neurocomputing, vol. 320, pp.195-202, 2018.

[12] C. Laske, H. R. Sohrabi, S. M. Frost, K. López-de-Ipiña, P. Garrard, M. Buscema, J. Dauwels, S.R. Soekadar, S. Mueller, C. Linnemann, and S.A., "Bridenbaugh, Innovative diagnostic tools for early detection of Alzheimer's disease," Alzheimer's \& Dementia, vol. 11, no. 5, pp. 561-578, 2015.

[13] G. A. Papakostas, A. Savio, M. Graña, and V. G. Kaburlasos, "A lattice computing approach to Alzheimer's disease computer assisted diagnosis based on MRI data," Neurocomputing", vol. 150, pp. 37-42, 2015.

[14] K. R. Kruthika, H. D. Maheshappa, and Alzheimer's "Disease Neuroimaging Initiative: CBIR system using Capsule Networks and 3D CNN for Alzheimer's disease diagnosis," Informatics in Medicine Unlocked, vol. 14, pp. 59-68, 2019.

[15] R. Ju, C. Hu, P. Zhou, and Q. Li, "Early diagnosis of Alzheimer's disease based on resting-state brain networks and deep learning," IEEE/ACM Transactions on Computational Biology and Bioinformatics (TCBB), vol. 16, no. 1, pp. 244-257, 2019.

[16] L. Yue, X. Gong, K. Chen, M. Mao, J. Li, A. K. Nandi, and M. Li, "Auto-Detection of Alzheimer's Disease Using Deep Convolutional Neural Networks," in 2018 14th International Conference on Natural Computation, Fuzzy Systems and Knowledge Discovery (ICNCFSKD), IEEE, pp. 228-234, July 2018. 